\title{
ANÁLISIS ESTRUCTURAL Y MORFOLÓGICO DE PELÍCULAS DE NITRURO DE ALUMINIO OBTENIDAS POR DEPOSICIÓN DE LÁSER PULSADO
}

\author{
STRUCTURAL AND MORPHOLOGICAL ANALYSIS OF ALUMINUM NITRIDE \\ FILMS OF OBTAINED BY PULSED LASER DEPOSITION
}

\author{
Jaime Andrés Pérez Taborda \\ Ing. Física, Universidad Tecnológica de Pereira, \\ Grupo plasma Láser y Aplicaciones \\ Pereira - Colombia, jaimeandres.perez@gmail.com \\ Henry Riascos Landázuri \\ Fisico, M.Sc., Ph.D., Departamento de Física, Universidad Tecnológica de Pereira, \\ Grupo plasma Láser y Aplicaciones \\ Pereira - Colombia, hriascos@utp.edu.co \\ Francy Nelly Jiménez García \\ Ing. Química, M.Sc. Ph.D., Universidad Autónoma de Manizales, \\ Departamento de Física y Matemática, Manizales - Colombia, \\ fnjimenezg@unal.edu.co \\ Julio César Caicedo Angulo \\ Ing. Materiales, Ph.D., Grupo películas delgadas, Universidad del Valle, \\ Cali - Colombia, icesarca@univalle.edu.co
}

Fecha de recepción: 27 de octubre de 2010

Fecha de aprobación: 30 de noviembre de 2010

\section{RESUMEN}

En este trabajo, se presentan los resultados preliminares de películas nanoestructuradas de nitruro de aluminio (AIN), que fueron depositadas con el método de deposición por láser pulsado (PLD). Al efecto, se utilizó un láser Nd:YAG $(\lambda=1064 \mathrm{~nm})$, que impacto un blanco de aluminio de alta pureza $(4 \mathrm{~N})$, en una atmósfera de nitrógeno. Se utilizaron como sustratos portaobjetos de vidrio, $\mathrm{Si}_{3} \mathrm{~N}_{4}(100)$ y $\mathrm{Si}(100)$. El tiempo de deposición fue de 15 minutos a una fluencia del láser $7 \mathrm{~J} / \mathrm{cm}^{2}$ y a temperatura ambiente. El espesor de las películas fue de $50 \mathrm{~nm}$ medido con un perfilómetro. Para estudiar la influencia del nitrógeno en las películas delgadas de AIN, se varió la presión del gas ambiente 
entre (3 y 4) mTorr. Igualmente se estudió la influencia del sustrato en las propiedades morfológicas de las películas delgadas de AIN. La nanoestructura de las películas se determinó mediante microscopia electrónica de barrido (SEM), y microscopia de fuerza atómica (AFM); la composición química, utilizando la técnica de espectroscopía de rayos $X$ por dispersión de energía (EDX). La estructura cristalina fue estudiada con difracción de rayos X (DRX), para la película de 4 mTorr sobre un sustrato de $\mathrm{Si}_{3} \mathrm{~N}_{4}(100)$, y se encontró una estructura policristalina con reflexiones de los planos (002), asociados a la estructura tipo wurtzita del AIN.

Palabras Clave: nitruro de aluminio (AIN), deposición por láser pulsado (PLD), DRX, SEM, EDX, perfilómetro, AFM.

\section{ABSTRACT}

This paper gives the preliminary results about aluminum nitride (AIN) nanoestructured films deposited by pulsed laser deposition (PLD) technique, by using laser Nd:YAG $(\lambda=1064)$, which hit a target of high purity aluminum $(4 \mathrm{~N})$ in a nitrogen atmosphere. We used glass slide, $\mathrm{Si}_{3} \mathrm{~N}_{4}(100)$ and $\mathrm{Si}(100)$ as substrates. The deposition time was 15 minutes at laser fluence $7 \mathrm{~J} / \mathrm{cm}^{2}$ and room temperature. The thicknesses of thin films were $50 \mathrm{~nm}$ measured with a profilometer. The influence of nitrogen on thin films was studied by changing room gas pressure between 3 and 4 mTorr. Also we have studied the influence of substrate on morphological properties of AIN thin films. The film nanostructure was determined by scanning electron microscopy (SEM), atomic force microscopy (AFM) -the chemical composition- using the technique of energy dispersive X-ray (EDX). The crystal structure was examined with X-Ray Diffraction (XRD) to a 4 mTorr film on a $\mathrm{Si}_{3} \mathrm{~N}_{4}$ (100) substrate giving a polycrystalline structure with reflections of planes (002), linked to the wurtzite-like structure of AIN.

Key words: aluminum nitride (AIN), pulsed laser deposition (PLD), XRD, SEM, EDX, profilometer, AFM.

\section{INTRODUCCIÓN}

En los últimos años, se ha realizado un esfuerzo importante en la búsqueda de nuevos materiales con estructuras cada vez más complejas, que presentan propiedades de conducción iónica y electrónicas. Entre los nuevos materiales estudiados, se destacan los nitruros metálicos que son un tipo de compuestos del cual no existe todavía un conocimiento profundo sobre los mecanismos de transporte de carga, por lo cual se hace necesario un estudio fundamental en muestras de gran calidad cristalina. 
El interés de obtener nanoestructuras de nitruro de aluminio en fase hexagonal por medio de la técnica de deposición por láser pulsado (PLD), se debe a su gran potencial de aplicaciones en LED, detectores en el azul y ultravioleta, inclusive en ambientes de alta temperatura [1], [2], [3]. El nitruro de aluminio cristaliza en el sistema hexagonal, con una estructura de tipo wurtzita (caracterizada por sus constantes de red a y $c$ ).

EL AIN es el material de banda más ancha a temperatura ambiente (Eg $=6,2 \mathrm{eV})$, y es considerado un semiconductor del grupo III-V [4]. El depósito por láser pulsado presenta algunas ventajas, especialmente en materiales formados por un blanco metálico (Al), en un medio de gas reactivo $\left(\mathrm{N}_{2}\right)$; además, se puede obtener películas con una buena calidad cristalina a baja temperatura del sustrato. El ánimo de este trabajo preliminar, es presentar películas nanoestructuradas de nitruro de aluminio (AIN), depositadas con el método de deposición por láser pulsado (PLD).

\section{MATERIALES Y METODOS}

Deposición por láser pulsado. Se depositaron dos conjuntos de películas de AIN por el método de Deposición por Láser Pulsado (PLD), a partir de un blanco de aluminio de alta pureza $(4 \mathrm{~N})$ de $2,54 \mathrm{~cm}$ de diámetro, $0,31 \mathrm{~cm}$ de espesor. El blanco se hizo girar a una frecuencia de $2,2 \mathrm{~Hz}$ con el fin de evitar la formación de cráteres y lograr una alta uniformidad en la deposición, la cual se llevó a cabo en un ambiente de nitrógeno de alta pureza $(5 \mathrm{~N})$, variando la presión de 3 mTorr a 4 mTorr. La deposición se hizo en una cámara de vacío que cuenta con una bomba mecánica seca modelo ACP 28 con una velocidad de evacuación de $140 \mathrm{l} / \mathrm{s}$ para el prevacío y una bomba turbomolecular Alcatel con una velocidad de bombeo $280 \mathrm{l} / \mathrm{s}$ para el alto vacío, que alcanzó una presión residual de $10^{-6} \mathrm{mTorr}$. Cada depósito constó de 9.000 pulsos de un láser Nd:YAG modelo INDI-30 Spectra Physics con $\lambda=1.064 \mathrm{~nm}$. La energía de los pulsos del láser fue de $500 \mathrm{~mJ}$ y una duración de $7 \mathrm{~ns}$, que impactaron el blanco en un ángulo de $45^{\circ}$ durante 15 minutos. La distancia blanco-sustrato fue de $5,4 \mathrm{~cm}$. Se utilizaron como sustratos portaobjetos de vidrio, $\mathrm{Si}_{3} \mathrm{~N}_{4}(100)$ y $\mathrm{Si}(100)$, que se limpiaron con metil-acetona en ultrasonido. La fluencia del láser se obtuvo, enfocando el haz del láser en el blanco mediante una lente de vidrio (con distancia focal de $24,5 \mathrm{~cm}$ ), que se mantuvo constante a $7 \mathrm{~J} / \mathrm{cm}^{2}$. La figura 1 muestra un esquema del montaje experimental, en trabajos previos se describen en detalle, el montaje y el procedimiento experimental [5]. 


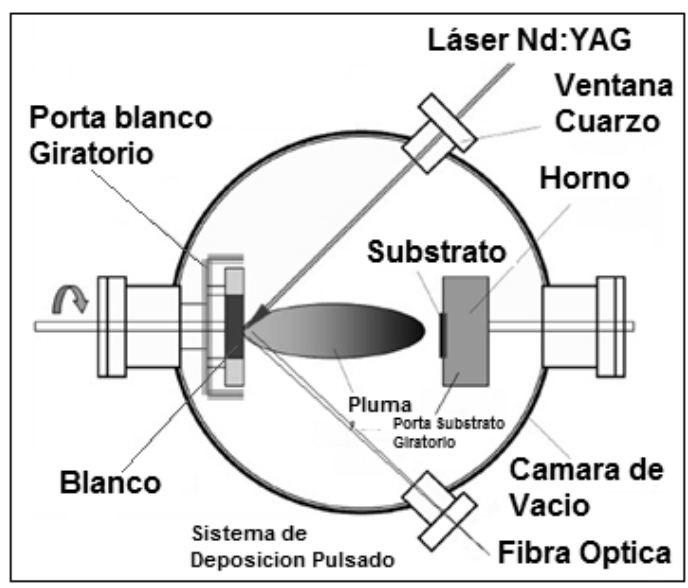

Figura 1. Esquema experimental utilizado para la deposición de películas delgadas de nitruro de aluminio por medio de PLD

Caracterización. El análisis morfológico de las películas se realizó con un microscopio electrónico de barrido SEM, en un equipo Jeol JSM-35 con una energía de $15 \mathrm{Kev}$, acompañado de un detector de rayos- $X$ y el detector de electrones secundarios de berilio-litio dentro de la cámara, con el propósito de amplificar la señal para el análisis en la espectroscopia de energía dispersiva de rayos-X (EDS). Se determinó el tamaño de grano y la rugosidad por medio de Microscopia de Fuerza Atómica (AFM), medidas realizadas en un microscopio de fuerza atómica Asylum Research MFP-3D-SA en el modo de contacto con un barrido de $2 \mu \mathrm{m} \times 2 \mu \mathrm{m}$. El espesor de las películas fue medido con un perfilómetro Dektak 8000 en el modo de corrido continuo de 0 a $7 \mathrm{~mm}$. La estructura cristalina de las películas se determinó por difracción de rayos-x, (DRX), usando un equipo SIEMENS D5000 con una configuración $\theta / 2 \theta$ y usando una fuente de Ka-Cu $(\lambda=1,5406 \AA)$.

\section{RESULTADOS Y DISCUSIÓN}

Análisis morfológico y estructural. Como se observa en la micrográfica de la Figura 2 , los cambios en la morfología del AIN dependen de la variación en la presión de depósito y sustrato. Esto es evidente pues para pequeñas variaciones de presión la calidad superficial de la película es afectada. En la figura de la micrografía 2(a) se observa que para una presión de 3 mTorr sobre un sustrato de $\mathrm{Si}$, la superficie de la película es homogénea, pero con un bajo contenido de nitrógeno debido a la baja presión de depósito. 
Tabla 1. Contribución en \% atómico obtenido por la técnica de EDX. Para la Imagen 2 (d), donde se observa el alto contenido de aluminio y la ausencia total de nitrógeno, características propias de un dropplet

\begin{tabular}{|l|c|}
\hline Elemento & $\begin{array}{c}\text { \% Atómico } \\
\mathbf{\mathbf { 0 . 1 0 }}\end{array}$ \\
\hline Al K & 60.79 \\
\hline Si K & 2.92 \\
\hline C K & 24.67 \\
\hline O K & 11.63 \\
\hline
\end{tabular}
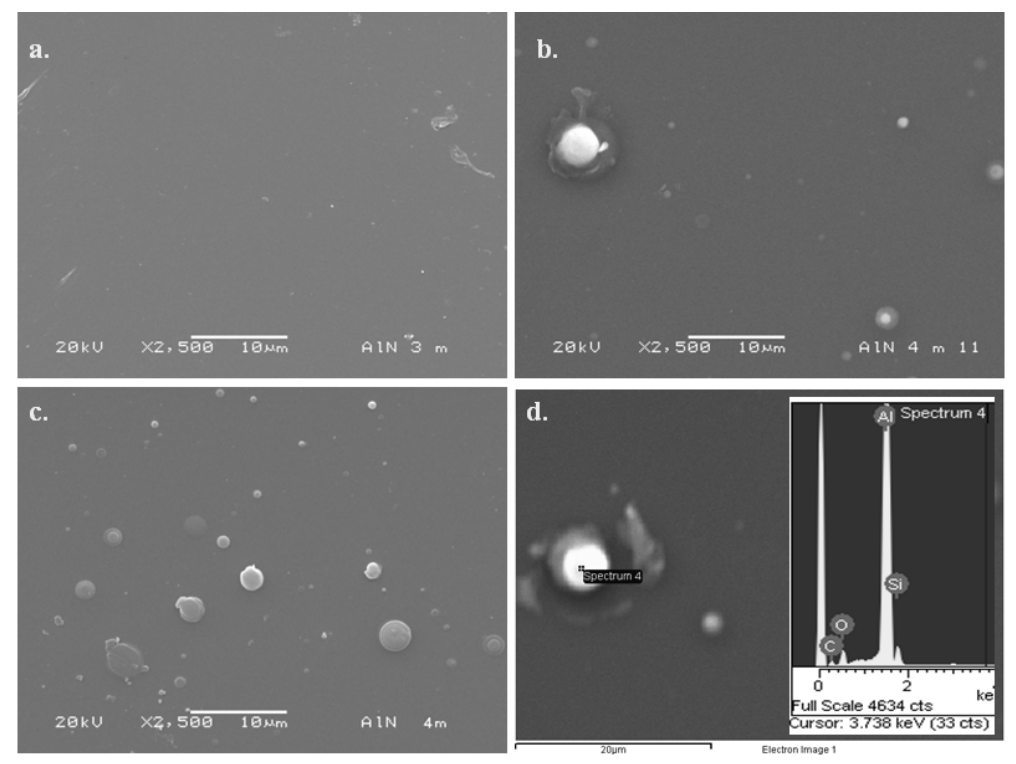

Figura 2. Imágenes SEM de películas delgadas de AIN depositadas por PLD a una fluencia del láser $7 \mathrm{~J} / \mathrm{cm}^{2}$ y a temperatura ambiente, con variaciones de presión y sustrato: (a) $3 \mathrm{mTorr}$ sobre sustrato de Si; (b) 4 mTorr sobre sustrato de $\mathrm{Si}_{3} \mathrm{~N}_{4}$; (c) 4 mTorr sobre porta-muestra de vidrio; (d) 4 mTorr sobre sustrato de $\mathrm{Si}_{3} \mathrm{~N}_{4}$, en la inserción la imagen EDX correspondiente

También se observan otras partículas con formas irregulares, lo cual puede ser atribuible a un proceso de exfoliación. Para una presión de $4 \mathrm{mTorr}$ sobre sustrato de $\mathrm{Si}_{3} \mathrm{~N}_{4}$, figura 2(b), se observa un crecimiento de tipo columnar, que favorece la formación de cráteres o de islas. Pero también observamos en la figura de las micrografías 2(b) y 2(c), la producción de gotas de ebullición o dropplets. Estas gotas son propias de la técnica de PLD y son ocasionadas por el desprendimiento de macropartículas que al impactar con el sustrato, generan este tipo de imperfecciones en la superficie de las películas [6]. Por medio de microscopia de fuerza atómica se complementó las medidas obtenidas por SEM.

La figura de la micrografía 2(d) muestra la composición química de la película crecida a una presión de 4 mTorr sobre sustrato de $\mathrm{Si}_{3} \mathrm{~N}_{4}$, en donde se observa para una región de la película, un alto contenido de aluminio $(60 \%)$, corroborando de esta manera la formación de dropplets (tabla 1). La calidad superficial y la orientación de la película dependen de la presión de nitrógeno y del sustrato utilizado. La baja presión del $\mathrm{N}_{2}$, aumenta el camino libre medio de las partículas constituyentes del plasma, lo cual en últimas, significa mayor energía cinética de las partículas que adhieren al sustrato. Esto y las posibles impurezas presentes en el sustrato, explican por qué la calidad superficial de la película crecida a 4 mTorr sobre portamuestra de vidrio no es buena, como se muestra en la micrografía 2(c).

Para la muestra crecida sobre $\mathrm{Si}_{3} \mathrm{~N}_{4}$ a una presión de 4 mTorr (figura 3), la rugosidad fue de 3,6 nm y el tamaño de grano de $62,9 \mathrm{~nm}$. Esto demuestra una buena calidad 
morfológica de las películas, aún al ser depositadas por la técnica de PLD que es un método altamente energético.

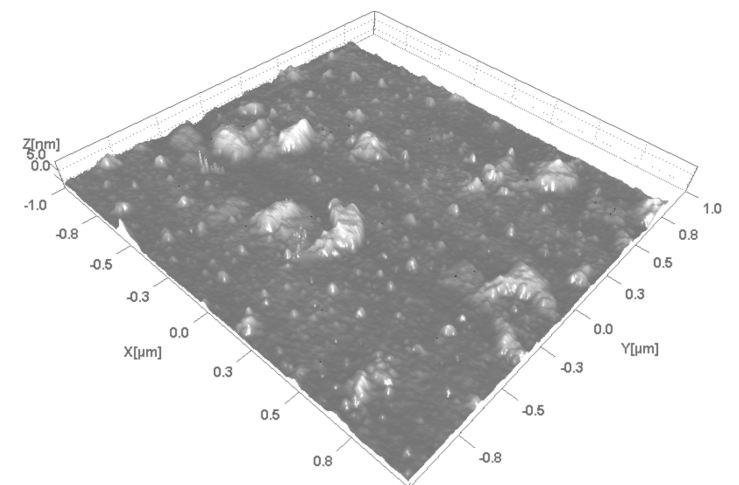

Figura 3. Microimagen AFM para una película de nitruro de aluminio crecida a $4 \mathrm{mTorr} \mathrm{sobre} \mathrm{un}$ sustrato de $\mathrm{Si}_{3} \mathrm{~N}_{4}$ con una fluencia del láser $7 \mathrm{~J} / \mathrm{cm}^{2}$, a temperatura ambiente La rugosidad fue de $3.6 \mathrm{~nm}$ y el tamaño de grano de $62.9 \mathrm{~nm}$

En la figura 4, se muestra el difractograma obtenido para una película de 4 mTorr sobre un sustrato de $\mathrm{Si}_{3} \mathrm{~N}_{4}$. En el difractograma, se observa que la película es policristalina, y presenta fases cúbicas [ $\alpha(200), \alpha(110)]$ y fases hexagonales [h (002), $h(006)]$ atribuibles a la formación de alúmina $\mathrm{Al}_{2} \mathrm{O}_{3}$. La fase hexagonal (002), corresponde a la estructura tipo wurtzita de las películas de AIN, que es la fase más estable de las estructuras posibles en los compuestos de AIN, debido a que se presenta acortamiento en la distancia entre los planos de la estructura, generando apilamiento en la fase zinc-blende [7], [8], [9].

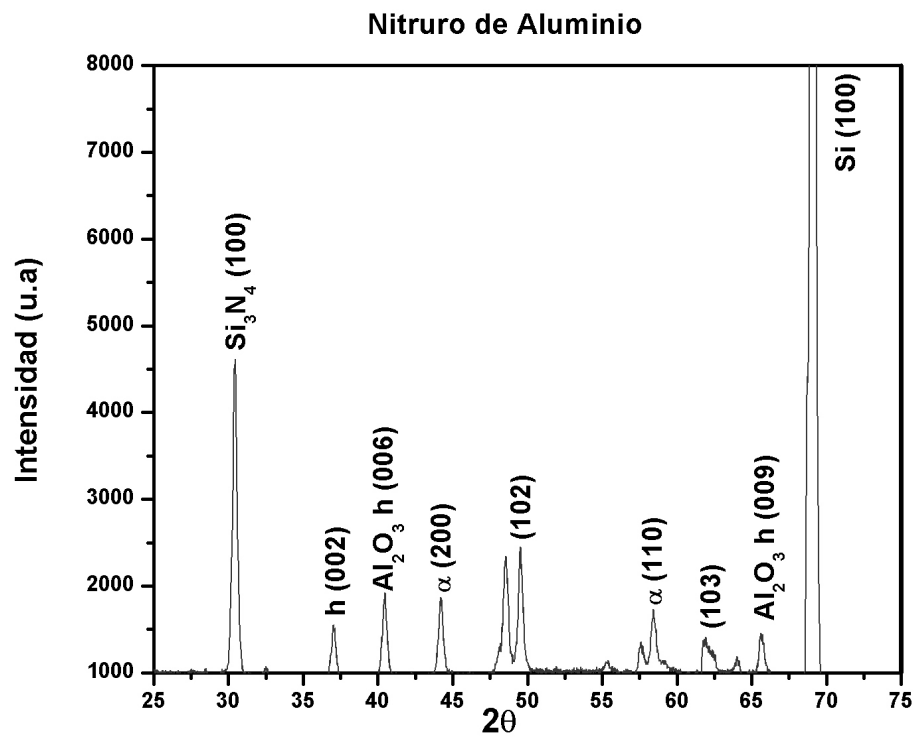

Figura 4. Difractograma obtenido para una película de nitruro de aluminio con una presión de nitrógeno de $4 \mathrm{mTorr}$ sobre un sustrato de $\mathrm{Si}_{3} \mathrm{~N}_{4}$, a una fluencia del láser $7 \mathrm{~J} / \mathrm{cm}^{2}$ y a temperatura ambiente 
Normalmente, se requiere calentar el sustrato para obtener cristalinidad en las películas. En este caso, la temperatura se logra por las altas velocidades de las especies presentes en el plasma $\left(\mathrm{Al}, \mathrm{N}_{2}\right)$, debido a la transferencia de energía durante el choque con el sustrato. Adicional a esto, el bajo espesor obtenido es un factor clave para las aplicaciones y propiedades de las películas de AIN (figura 5).

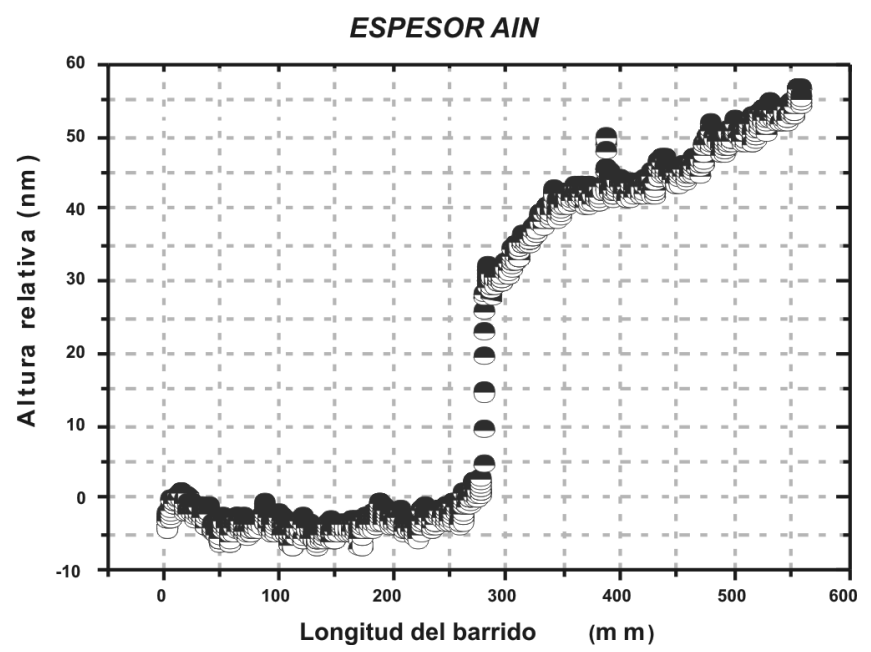

Figura 5. Medida obtenida en el perfilómetro para una muestra de AIN crecida a una presión de 4 mTorr en un sustrato de $\mathrm{Si}_{3} \mathrm{~N}_{4}$ preparada con un escalón mediante una máscara de vidrio, la altura del escalón fue de $\sim 50 \mathrm{~nm}$

Para los plasmas que se producen por láseres con pulsos de duración de nanosegundos, como en este caso, el calentamiento se da durante algunos microsegundos sólo en las capas más superiores. El máximo incremento de temperatura es casi lineal con la energía cinética media de las partículas incidentes. Se ha estudiado que iones con energías mayores que $100 \mathrm{eV}$, producen en la superficie del sustrato, una capa fina ( algunas decenas de $\AA$ ), de vacancias individuales que se difunden profundamente dentro del sustrato, y se recombinan en un tiempo $\sim 10^{-8} \mathrm{~s}$, sin afectar en gran medida la cristalinidad [10]. También se debe tomar en cuenta que el máximo de elevación de la temperatura de la superficie del sustrato es aproximadamente proporcional a $1 / \mathrm{d}^{2}$, donde d es la distancia del blanco al sustrato [9[, [10], [11].

\section{CONCLUSIONES}

Se logró crecer mediante deposición por láser pulsado, películas delgadas de AIN, utilizando un láser pulsado de Nd:YAG. Se observa una nanoestructura policristalina en las películas crecidas a temperatura ambiente y a bajas presiones del gas de trabajo, y queda de manifiesto que la técnica es beneficiosa para este tipo de crecimientos debido en especial, a las altas energías de las partículas de la pluma que se depositan sobre 
el sustrato, aún para una separación relativamente grande entre el blanco sustrato como es en este caso.

La calidad morfológica de las películas es buena, en especial para las películas crecidas sobre los sustratos de $\mathrm{Si}(100)$ y $\mathrm{Si}_{3} \mathrm{~N}_{4}(100)$. Para las películas crecidas sobre sustrato de portaobjetos de vidrio, se observan partículas con formas irregulares atribuibles a formación de dropplets, impurezas del sustrato o procesos de exfoliación.

Se logra identificar dropplets que son típicos de la técnica, al igual que un crecimiento de tipo columnar. El análisis por AFM indica que el tamaño de grano es del orden de los manómetros, y posee una baja rugosidad. El espesor de las películas es del orden de nanómetros lo cual es conveniente para las futuras aplicaciones de las películas en dispositivos optoelectrónicos.

\section{REFERENCIAS BIBLIOGRÁFICAS}

[1] M. A. Dubois (1999), Aluminium nitride and lead zirconate-titanate thin films for ultrasonic applications: Integration, properties and devices, Thesis doctoral, Département des Matériaux, Ecole Polytechnique Fédérale de Lausanne (Suiza).

[2] H. Harima, J. (2002), Phys.: Condens. Matter 14, pp. R967-R993.

[3] G. F. Iriarte, (2003) AIN Thin Film Electroacoustic Devices. Acta Universitatis Upsaliensis, Uppsala Universitet.

[4] Vurghaftman, J. R. Meyer and L. R. Ram- Mohan, J. (2001), Appl. Physics 89, pp.5815- 5875.

[5] H. Riascos, J. Niedhardt, G. Radnoczi, J. Emmerlich, G. Zambrano, L. Hultman, P. Prieto. (2004), Thin Solid Films 497, p. 1-6.

[6] S. Metev, (1994), Pulsed Laser Deposition of Thin Films, edited by B.C. Douglas and K.H. Graham, John Wiley \& Sons, NY, pp. 255.

[7] H. Yoshiyama, S. Tanaka, Y. Mikami, S. Ohshio, J. Nishiura, H. Kawakami, H. Kobayashi, J. Cryst. (1988), Growth 86, p. 56-61.

[8] N. Fujimura, T. Nishihara, S. Goto, J. Xu, T. Ito, J. Cryst. (1993), Growth 130 p.269. 
[9] S. Bakalova, A. Szekeres, A. Cziraki, C.P. Lungu, S. Grigorescu, G. Socol, E. Axente, I.N. Mihailescu, (2007) Appl. Surf. Sci. 253 pp. 8215-8218

[10] C.D. Wagner, W.M. Riggs, L.E. Davis, J.F. Moulder, G.E. Muilenberg (1979), Handbook of X-ray Photoelectron Spectroscopy, Physical Electronics, Eden Prairie, Minnesota.

[11] X. Xu, J. Appl. (1995), Phys. 77, pp.6715-6719. 
